\title{
Polarons in magnesium-doped lithium niobate crystals induced by femtosecond light pulses
}

\author{
Dominik Maxein • Stephan Kratz • Peter Reckenthaeler • Johanna Bückers • \\ Daniel Haertle • Theo Woike • Karsten Buse
}

Received: 9 June 2008 / Published online: 26 July 2008

(C) The Author(s) 2008. This article is published with open access at Springerlink.com

\begin{abstract}
Strong light-induced absorption has been observed in lithium niobate crystals doped with magnesium after application of femtosecond illumination. In this material there are no $\mathrm{Nb}$-on-Li-site defects and hence no antisite polarons occur, but small free polarons close to the conduction band can be generated. The light-induced absorption observed is attributed to these polarons. For $\mathrm{LiNbO}_{3}: \mathrm{Mg}$, their decay times are about two orders of magnitude smaller than those of the $\mathrm{Nb}$-on-Li-site polarons in undoped material. The results are relevant for a better understanding of the suppression of the so-called optical damage in these crystals and for their use in femtosecond applications.
\end{abstract}

PACS $42.70 . \mathrm{Mp} \cdot 71.38 . \mathrm{k} \cdot$ 42.50.Gy

\section{Introduction}

Lithium niobate crystals $\left(\mathrm{LiNbO}_{3}\right)$ are very attractive for nonlinear-optical applications, in particular because periodic poling of this material enables quasi-phase-matching [1].

D. Maxein $(\bowtie) \cdot S$. Kratz $\cdot$ P. Reckenthaeler · J. Bückers .

D. Haertle $\cdot$ K. Buse

Physikalisches Institut, Universität Bonn, Wegelerstraße 8,

53115 Bonn, Germany

e-mail: maxein@physik.uni-bonn.de

url: http://www.photonik.uni-bonn.de/

P. Reckenthaeler

Max-Planck-Institut für Quantenoptik, Hans-Kopfermann-

Straße 1, 85748 Garching, Germany

T. Woike

Institut für Mineralogie, Universität zu Köln, Zülpicherstr. 49b,

50674 Köln, Germany
A serious obstacle is, however, the so-called optical damage, i.e., light-induced refractive index changes [2]. Besides other methods, it has been shown that optical damage can be suppressed effectively by doping $\mathrm{LiNbO}_{3}$ with magnesium $[3,4]$, thus making $\mathrm{LiNbO}_{3}: \mathrm{Mg}$ the material of choice for various applications [5]. However, since the peak intensities used tend to become higher and higher, e.g., in intracavity optical parametric oscillators or ultrashort pulse applications, the need for a deeper understanding of this effect, its limits, and its potential for improvement becomes more demanding.

The existence of small bound polarons at niobium-onlithium site $\left(\mathrm{Nb}_{\mathrm{Li}}^{4+/ 5+}\right.$ "antisite defect") in congruently melting $\mathrm{LiNbO}_{3}$ supports the occurrence of optical damage [6]. In $\mathrm{LiNbO}_{3}$ doped with a sufficient amount of $\mathrm{Mg}$, the formation of these states is suppressed due to the disappearance of the antisite defects $[7,8]$; small free polarons at niobiumon-niobium site $\left(\mathrm{Nb}_{\mathrm{Nb}}^{4+/ 5+}\right)$ play the major role in this case $[9,10]$.

To learn more about the physical mechanisms, we employed femtosecond light pulses to investigate the lightinduced absorption, both in $\mathrm{Mg}$-doped and undoped $\mathrm{LiNbO}_{3}$. Similar experiments with nanosecond pulses have been reported by Conradi et al. [11]. However, fs pulses have a higher peak intensity and a duration much shorter than the recombination timescale of the excited electrons, so a welldefined initial state after illumination can be generated. The decay of the light-induced absorption due to polarons can be well described by a Kohlrausch-Williams-Watts (KWW) (or so-called "stretched exponential") function [12-14]:

$\alpha_{\mathrm{li}}=\alpha_{\mathrm{li}, \mathrm{Plat}} \exp \left[-(t / \tau)^{\beta}\right]$.

Here, $\alpha_{1 \mathrm{i}}$ is the light-induced absorption, and $\alpha_{\mathrm{li} \text {,Plat }}$ is its initial value, i.e., the light-induced absorption immediately 
after the illumination with the pump light. Finally, $\tau$ is the decay time constant, and $\beta$ is the stretching coefficient.

\section{Experimental setup}

Two samples are used in the experiments: An undoped lithium niobate crystal (short LNU) from Crystal Technologies, Inc. and a lithium niobate crystal doped with $5 \mathrm{~mol} \%$ $\mathrm{MgO}$ (short LNMg) from Yamaju Ceramics. Both samples are congruently melting and $x$-cut, their thickness is $80 \mu \mathrm{m}$ each.

Every ten seconds (unless noted otherwise) a femtosecond pulse of $1 \mathrm{~mJ}$ with a wavelength of $776 \mathrm{~nm}$ originates from a Clark-MXR CPA2010 laser system. Either a small fraction of this pulse is separated using a beam splitter, or the pulse is used to generate a pulse of $1310 \mathrm{~nm}$ wavelength via optical parametric amplification (OPA) in a Light Conversion, Inc. TOPAS system. The remaining fundamental pulse is frequency doubled in a BBO crystal to a wavelength of $388 \mathrm{~nm}$. All pulses have a full-width-at-half-maximum (FWHM) pulse duration of about $200 \mathrm{fs}$. The $388-\mathrm{nm}$ pulses act as pump light to generate light-induced absorption, while the IR pulses of 776 or $1310 \mathrm{~nm}$ are attenuated and used to probe these absorption changes. The probe pulses can be delayed with respect to the pump pulses via a delay stage. In addition, continuous-wave (cw) lasers with the wavelengths $532,780,980,1064,1310$, and $1532 \mathrm{~nm}$ are available as probe light sources. The probe light can be polarized parallel (extraordinarily polarized, e-pol) or perpendicular to the crystal's $c$-axis (ordinarily polarized, o-pol). The pump light is e-pol, unless noted otherwise.

Pump beam and probe beam are superimposed using a dielectric mirror. From there they propagate collinearly (angle difference $\lesssim 1 / 60 \mathrm{rad}$ ) through the sample and through a pinhole of $0.5 \mathrm{~mm}$ diameter onto which the crystal is mounted. The pump beam diameter (FWHM) at the crystal is about $1 \mathrm{~mm}$. All probe beams are even larger, so we assume a homogeneous illumination of the sample volume that is observed through the pinhole. Behind the crystal holder, the pump light is blocked via a dichroic mirror and edge filters, while the probe light is focussed onto a photodiode. In the case of pulsed probe light, the probe pulse energy after passing through the crystal is measured as a function of the delay between pump and probe, i.e., the delay-stage position. For $\mathrm{cw}$ probe light, the temporal evolution of the transmitted power is recorded directly with an oscilloscope. The former measurement has a time resolution given approximately by the probe pulse duration, and the maximum possible delay is $6 \mathrm{~ns}$. The latter method is limited by the bandwidth of the photodiode $(200 \mathrm{MHz})$, therefore events faster than $5 \mathrm{~ns}$ can not be resolved with $\mathrm{cw}$ probe light.

\section{Results}

Figures 1 and 2 show the generation and the decay of the light-induced absorption $\alpha_{\mathrm{li}}$ for LNU and LNMg for probe wavelengths of $776 / 780 \mathrm{~nm}$ and $1310 \mathrm{~nm}$, respectively. The plots are composed of measurements with probe pulses for $t<6 \mathrm{~ns}$ and $\mathrm{cw}$ light for $t \geq 6 \mathrm{~ns}$. To fit the two parts together, the $\mathrm{cw}$ results had to be scaled with a factor of 1.2 for Fig. 2, which is within the precision of our measurements.

All four curves have a sharp rise at $t=0$, the time of the fs pulse illumination, and after less than 1 ps they drop to a plateau value, where $\alpha_{\mathrm{li}}$ is almost constant up to about $1 \mathrm{~ns}$. Then $\alpha_{\text {li }}$ decays to zero on a timescale of several nano- to milliseconds. For both probe wavelengths, the peak values of the light-induced absorption are almost identical for both samples, and the decay of $\alpha_{\mathrm{li}}$ is much faster in LNMg than in LNU. Probing with $776 \mathrm{~nm}$ o-pol light (Fig. 1) leads to similar plateau values of $\alpha_{\mathrm{li}}$ for both samples, but with e-pol light at a probe wavelength of $1310 \mathrm{~nm}$, the effect for LNU is about a factor of thirteen lower than that for LNMg. For an intermediate wavelength of $980 \mathrm{~nm}$, the plateau values of both samples differ by a factor of 2 for o-pol and 6 for
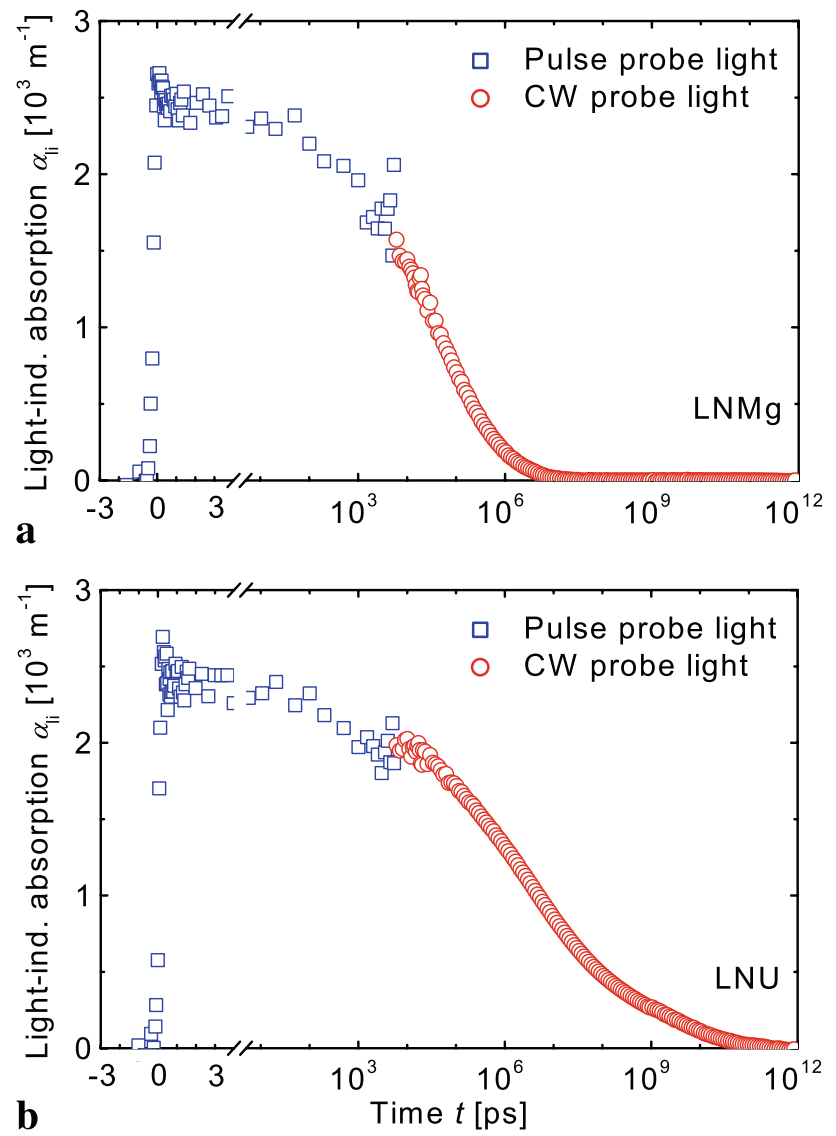

Fig. 1 Time dependence for the light-induced absorption with a pump pulse fluence $\Phi_{\text {Pulse }} \approx 1200 \mathrm{Jm}^{-2}$ at a repetition rate of $1 \mathrm{~Hz}$ a in $\mathrm{LNMg}$ and $\mathbf{b}$ in LNU. Time is set to zero at the maximum of $\alpha_{\mathrm{li}}$. The probe wavelength is $780 \mathrm{~nm}$, and the probe polarization is o-pol 


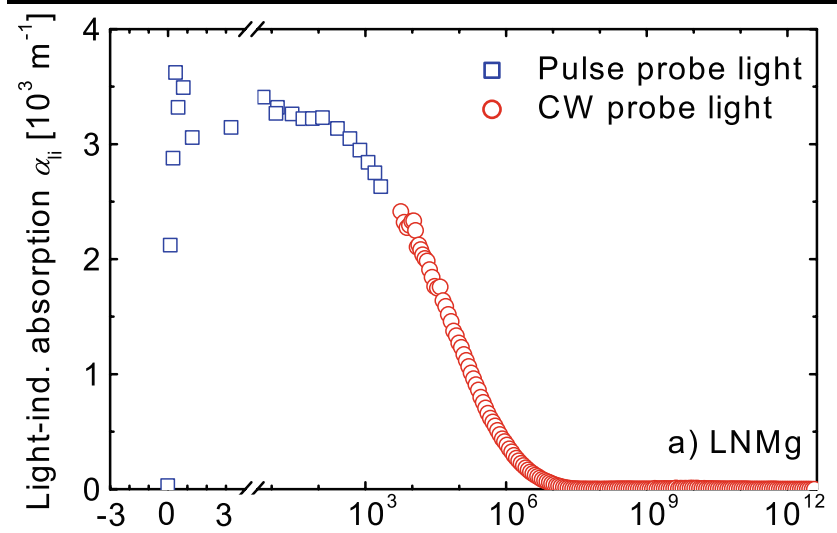

$\mathbf{a}$

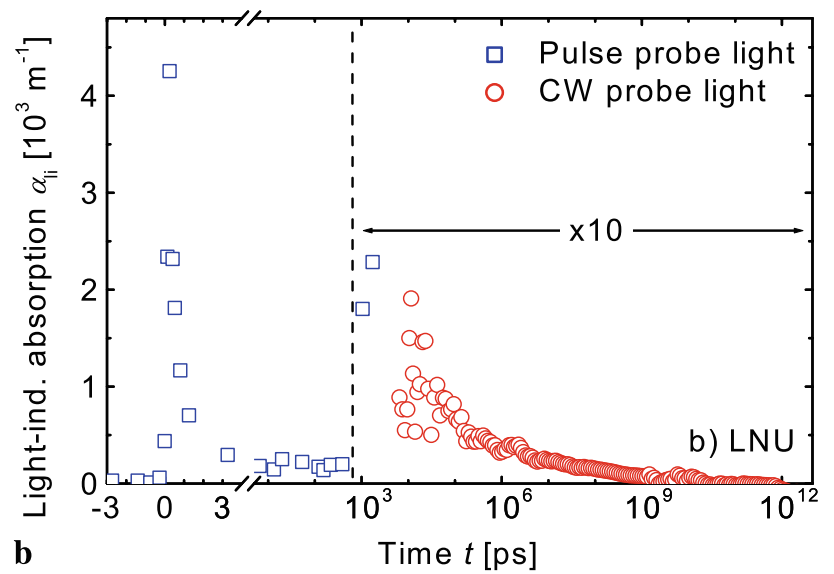

Fig. 2 Temporal evolution of the light-induced absorption with a pump pulse fluence $\Phi_{\text {Pulse }} \approx 640 \mathrm{Jm}^{-2} \mathbf{a}$ in LNMg and $\mathbf{b}$ in LNU. Time is set to zero at the maximum of $\alpha_{\mathrm{li}}$. The probe wavelength is $1310 \mathrm{~nm}$, the probe polarization is e-pol

e-pol probe light: The dependence on the probe polarization is for $\mathrm{LNU}$ just opposite to that of $\mathrm{LNMg}$, as is evident from Fig. 3. Here, the plateau values are plotted as a function of the pump pulse fluence $\Phi_{\text {Pulse }}$. The values originate from a fit of (1) to the data obtained with the $980 \mathrm{~nm} \mathrm{cw}$ probe laser. Within the measurement accuracy, a slightly super-linear increase is observed in all cases.

The dependence of the plateau value $\alpha_{\mathrm{li}, \text { Plat }}$ in $\mathrm{LNMg}$ on the probe wavelength was measured with several $\mathrm{cw}$ probe lasers: In Fig. 4, the light-induced absorption $10 \mathrm{~ns}$ after the pump pulse illumination is plotted versus the probe wavelength. For e-pol probe light, the effect has its maximum around $1300 \mathrm{~nm}$ and decreases about a factor of 4 towards $500 \mathrm{~nm}$. In the case of o-pol probe light, the behavior is qualitatively the same.

Resulting from $\mathrm{cw}$ probe measurements with 980 and $1310 \mathrm{~nm}$, we get decay times of $\alpha_{\mathrm{li}}$ as a function of the pulse fluence $\Phi_{\text {Pulse }}$, shown in Fig. 5. As also noted in [15] for undoped lithium niobate crystals, we observe a decrease of $\tau$ with increasing pump pulse fluence, both for LNU and for $\mathrm{LNMg}$. However, here the main observation is a two orders

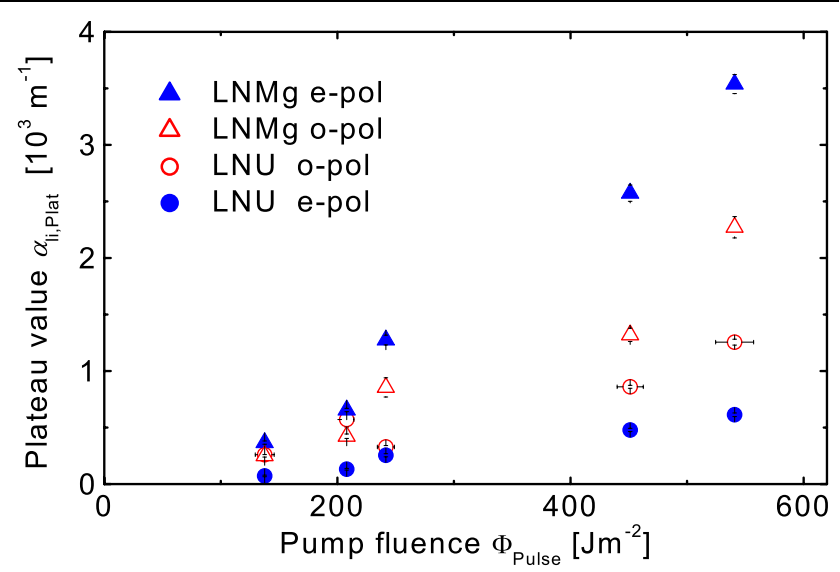

Fig. 3 Plateau amplitudes $\alpha_{\mathrm{li}, \text { Plat }}$ of the light-induced absorption for LNMg and LNU as a function of the pump pulse fluence $\Phi_{\text {Pulse }}$ for different probe polarizations. The $\mathrm{cw}$ probe light has a wavelength of $980 \mathrm{~nm}$. For $\mathrm{LNMg}$, the pump light is o-pol

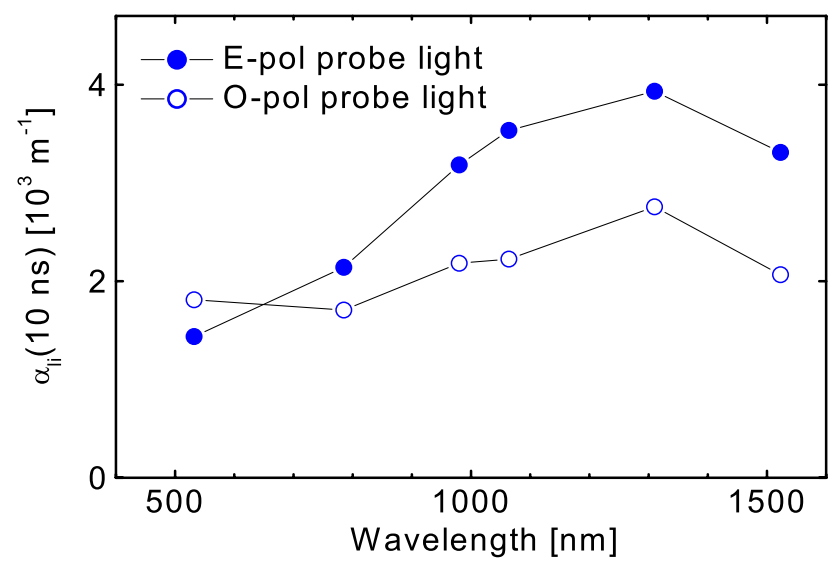

Fig. 4 Dependence of the light-induced absorption $\alpha_{\mathrm{li}}$ of LNMg on the $\mathrm{cw}$ probe wavelength. Here $\alpha_{\mathrm{li}}$ is measured $10 \mathrm{~ns}$ after the pump pulse, at a pump pulse fluence of $\Phi_{\text {Pulse }} \approx 1900 \mathrm{Jm}^{-2}$. The lines are guides to the eye

of magnitude smaller decay time constant for LNMg than that for LNU for all $\Phi_{\text {Pulse }}$, i.e., the decay of $\alpha_{\text {li }}$ happens much faster in LNMg. Finally, another important quantity obtained by the fits is the stretching coefficient $\beta$, shown in Fig. 6. While $\beta$ is constant in LNU at a value of about $0.12 \pm 0.05, \beta$ is larger in $\mathrm{LNMg}$ but decreases with rising pump pulse fluence.

\section{Discussion}

The feature first attracting attention is the very strong lightinduced absorption in LNMg: It is much stronger than in LNU by a factor of 3 for $980 \mathrm{~nm}$ o-pol probe light (Fig. 3) and by a factor of more than 10 for $1310 \mathrm{~nm}$ e-pol probe light (Fig. 2). In [15], the light-induced absorption in undoped $\mathrm{LiNbO}_{3}$ was reported to decrease between 776 and 


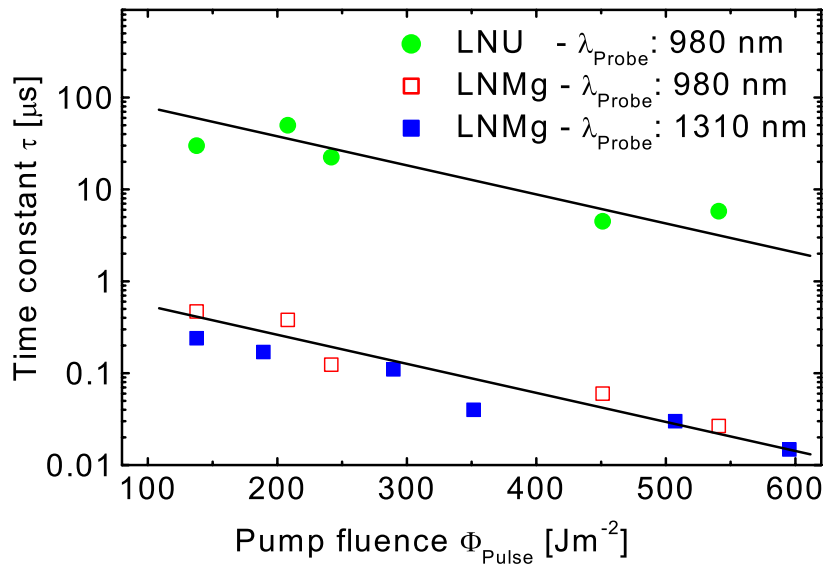

Fig. 5 Decay time constant of the light-induced absorption $\alpha_{\mathrm{li}}$ in LNU and $\mathrm{LNMg}$ as a function of the pump pulse fluence $\Phi_{\text {Pulse }}$. For LNMg, results for two different probe wavelengths are shown

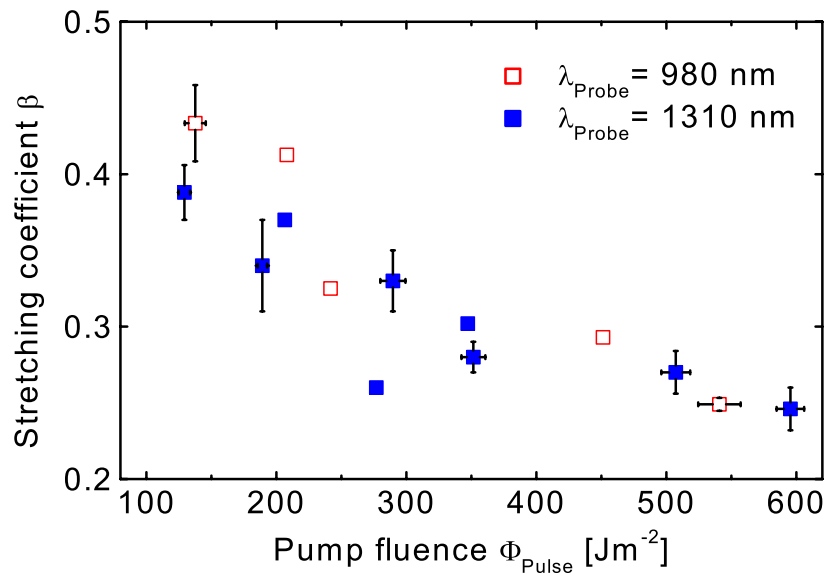

Fig. 6 Stretching coefficient $\beta$ of the decay of the light-induced absorption $\alpha_{\text {li }}$ vs. the pump pulse fluence $\Phi_{\text {Pulse }}$ in $\mathrm{LNMg}$

$1500 \mathrm{~nm}$; in contrast, in LNMg we observe an increase of $\alpha_{\mathrm{li}}$ from $532 \mathrm{~nm}$ to a maximum at $1310 \mathrm{~nm}$ (Fig. 4). This spectral dependence of $\alpha_{\mathrm{li}}$ in LNMg matches the absorption of small free polarons at $\mathrm{Nb}_{\mathrm{Nb}}^{4+/ 5+}$ sites observed in reduced $\mathrm{Mg}$-doped $\mathrm{LiNbO}_{3}$ [16]. Additionally, the dependence of $\alpha_{\mathrm{li}}$ on the probe polarization in LNMg is the same as the dependence of the absorption coefficient on the light polarization reported for small free polarons in [9]. Therefore, the large light-induced absorption observed after fs illumination of LNMg can be attributed to small free polarons created after excitation of electrons by two-photon absorption. They have an absorption maximum at about $1300 \mathrm{~nm}$, while in LNU small bound polarons are dominant, with a maximum absorption at $\approx 800 \mathrm{~nm}$ [9]. Also the much faster recombination times in LNMg with respect to those in LNU support this interpretation: Small polarons at $\mathrm{Nb}_{\mathrm{Nb}}$ are trapped less strongly than those at $\mathrm{Nb}_{\mathrm{Li}}$, making them more mobile and hence reducing the hopping time.
An interesting additional fact is the decrease of the coefficient $\beta$ in $\mathrm{LNMg}$ with increasing pump pulse fluence. According to [17], assuming hopping transport, $\beta$ is a function of two parameters: The ratio $\bar{R} / a$ between the average distance $\bar{R}$ covered in one hop by the polarons and the radius $a$ of the polaron, and the ratio $N_{0} / N_{\mathrm{T}}$ between the number of transport sites $N_{0}$ and the available trap sites $N_{\mathrm{T}}$. For low intensities, the latter are mainly defect sites which can accept electrons. However, with every electron, which we excite out of the valence band, also a hole is generated. With increasing intensity, their number also increases, and they become more and more important for recombination. In the regime where the majority of the traps are holes, $N_{\mathrm{T}}$ increases together with the number of electrons as a function of intensity, leading to the observed decrease of the stretching coefficient $\beta$. One might argue that this is also true for LNU. However, the polarons in LNU and LNMg are of different nature, and the other parameters governing $\beta$, namely the hopping distance $\bar{R}$, the polaron radius $a$, and the transport site density $N_{0}$, are different, leading to an overall smaller $\beta$ in LNU. Since in LNU $\beta$ is much closer to its limit 0 , the dependence on $N_{\mathrm{T}}$ is much weaker than in LNMg.

The photons of the 388-nm pump light used in this study are sufficient for two-photon band-to-band excitations. However, at sufficient intensities, the same effects can be expected for longer pump wavelengths because of multiplephoton excitations [18]. Thus, also in $\mathrm{Mg}$-doped $\mathrm{LiNbO}_{3}$, the light induced absorption sets an upper intensity limit; above this, the crystals cease to operate, because they become intransparent for infrared light.

\section{Conclusions}

We investigated with different probe wavelengths between 532 and $1532 \mathrm{~nm}$ the light-induced absorption in LNU and $\mathrm{LNMg}$ after fs illumination. The induced absorption is much higher in LNMg than in LNU around $1300 \mathrm{~nm}$. This has to be taken into account when using LNMg in nonlinear optics with high light intensities. The absorption changes are attributed to small free polarons at $\mathrm{Nb}_{\mathrm{Nb}}^{4+/ 5+}$ sites. The intensity dependence of the stretching coefficient $\beta$ of the KWWshaped $\alpha_{\text {li }}$ decay indicates that holes generated by the twophoton excitation of electrons from the valence band are the main annihilation sites for polarons in the regime of our experiments.

Acknowledgements Financial support from the DFG (award BU 913/19-1) and from the Deutsche Telekom AG is gratefully acknowledged.

Open Access This article is distributed under the terms of the Creative Commons Attribution Noncommercial License which permits any noncommercial use, distribution, and reproduction in any medium, provided the original author(s) and source are credited. 


\section{References}

1. L.E. Myers, R.C. Eckardt, M.M. Fejer, R.L. Byer, W.R. Bosenberg, J.W. Pierce, J. Opt. Soc. Am. B 12, 2102 (1995)

2. A. Ashkin, G.D. Boyd, J.M. Dziedzic, R.G. Smith, A.A. Ballman, O. Levinstein, K. Nassau, Appl. Phys. Lett. 9, 72 (1966)

3. G.G. Zhong, J. Jin, Z.K. Wu, J. Opt. Soc. Am. 70, 631 (1980)

4. D.A. Bryan, R. Gerson, H.E. Tomaschke, Appl. Phys. Lett. 44, 847 (1984)

5. L. Arizmendi, Phys. Stat. Sol. A 201, 253 (2004)

6. F. Jermann, J. Otten, J. Opt. Soc. Am. B 10, 2085 (1993)

7. B.C. Grabmaier, W. Wersing, W. Koestler, J. Cryst. Growth 110, $339(1991)$

8. U. Schlarb, K. Betzler, Phys. Rev. B 50, 751 (1994)

9. B. Faust, H. Müller, O.F. Schirmer, Ferroelectrics 153, 297 (1994)

10. G.K. Kitaeva, K.A. Kuznetsov, A.N. Penin, A.V. Shepelev, Phys. Rev. B 65, 054304 (2002)

11. D. Conradi, C. Merschjann, B. Schoke, M. Imlau, Capter MB3, in Controlling Light with Light: Photorefractive Effects, Photosensi- tivity, Fiber Gratings, Photonic Materials and More on CD-ROM (The Optical Society of America, Washington, 2007)

12. D. Berben, K. Buse, S. Wevering, P. Herth, M. Imlau, T. Woike, J. Appl. Phys. 87, 1034 (2000)

13. P. Herth, D. Schaniel, Th. Woike, T. Granzow, M. Imlau, E. Krätzig, Phys. Rev. B 71, 125128 (2005)

14. C. Merschjann, D. Berben, M. Imlau, M. Wöhlecke, Phys. Rev. Lett. 96, 186404 (2006)

15. O. Beyer, D. Maxein, T. Woike, K. Buse, Appl. Phys. B 83, 527 (2006)

16. G.K. Kitaeva, K.A. Kuznetsov, V.F. Morozova, I.I. Naumova, A.N. Penin, A.V. Shepelev, A.V. Viskovatich, D.M. Zhigunov, Appl. Phys. B 78, 759 (2004)

17. B. Sturman, E. Podivilov, M. Gorkunov, Phys. Rev. Lett. 91, $176602(2003)$

18. O. Beyer, D. Maxein, K. Buse, B. Sturman, H.T. Hsieh, D. Psaltis, Phys. Rev. E 71, 056603 (2005) 